\title{
CERTAIN HOMOGENEOUS UNICOHERENT INDECOMPOSABLE CONTINUA
}

\author{
F. BURTON JONES
}

A simple closed curve is the simplest example of a compact, nondegenerate, homogeneous continuum. If a bounded, nondegenerate, homogeneous plane continuum has any local connectedness property, even of the weakest sort, it is known to be a simple closed curve $[1,2,3] .{ }^{1}$ The recent discovery of a bounded, nondegenerate, homogenous plane continuum which does not separate the plane $[4,5]$ has given substance to the old question as to whether or not such a continuum must be indecomposable. Under certain conditions such a continuum must contain an indecomposable continuum [6]. It is the main purpose of this paper to show that every bounded homogeneous plane continuum which does not separate the plane is indecomposable.

Notation. If $M$ is a continuum and $x$ is a point of $M, U_{x}$ will be used to denote the set of all points $z$ of $M$ such that $M$ is aposyndetic at $z$ with respect to $x .^{2}$ It is evident that $U_{x}$ is an open subset of $M$.

Lemma. If the compact metric continuum $M$ is homogeneous and $x$ and $y$ are distinct points of $M$, then $U_{y}$ is not a proper subset of $U_{x}$.

Proof. Suppose on the contrary that $U_{y}$ is a proper subset of $U_{x}$. Since $M$ is homogeneous, there exists a homeomorphism $T$ such that $T(M)=M$ and $T(x)=y$. Then $T\left(U_{x}\right)=U_{y}$ and $T\left(U_{y}\right)$ is $U_{T(y)}$ which is a proper subset of $U_{y}$. Hence there exists a sequence $x_{0}=x, x_{1}=y$, $x_{2}=T(y), \cdots, x_{n}=T^{n}(x), \cdots$ of points of $M$ such that for each positive integer $n, U_{x_{n}}$ is a proper subset of $U_{x_{n-1}}$. For no two nonnegative integers $i$ and $j$ is $x_{i}=x_{j}$, because if $x_{i}=x_{j}$ then $U_{x_{i}}=U_{x_{j}}$. Consequently the sequence $x_{1}, x_{2}, x_{3}, \cdots$ has a limit point $x_{\omega}$. Now for each positive integer $n, U_{x_{\omega}}$ is a subset of $U_{x_{n}}$, because if $p$ is a point of $U_{x_{\omega}}$ there exist a subcontinuum $K$ of $M$ and an open subset $V$ of $M$ such that $M-x_{\omega} \supset K \supset V \supset p$; hence for infinitely many positive integers $n, M-x_{n} \supset K \supset V \supset p$; so for infinitely many positive integers $n, M$ is aposyndetic at $p$ with respect to $x_{n}$ and hence $p$ belongs to $U_{x_{n}}$.

Evidently $x_{\omega} \neq x_{n}, n=1,2,3, \cdots$. And since $M$ is homogeneous,

Presented to the Society, December 28, 1950; received by the editors December 26, 1950.

${ }^{1}$ Numbers in brackets refer to the bibliography at the end of this paper.

2 The continuum $M$ is aposyndetic at the point $z$ of $M$ with respect to the point $x$ of $M$ provided that $M$ contains a continuum $K$ and an open (rel. $M$ ) subset $V$ such that $M-x \supset K \supset V \supset$. 
there exists a homeomorphism $T_{1}$ such that $T_{1}(M)=M$ and $T_{1}(x)$ $=x_{\omega}$. Then $T_{1} T T_{1}^{-1}$ is a homeomorphism of $M$ onto itself such that if we let $x_{\omega+1}=T_{1} T T_{1}^{-1}\left(x_{\omega}\right), T_{1} T T_{1}^{-1}\left(U_{x_{\omega}}\right)=U_{x_{\omega+1}}$ which is a proper subset of $U_{x_{\omega}}$. This process can be continued uncountably many times to produce a well-ordered sequence $\alpha=x_{1}, x_{2}, x_{3}, \cdots, x_{i}, \cdots\left(i<\omega_{1}\right)$, of distinct points of $M$ such that (1) if $x_{i}$ of $\alpha$ has no immediate predecessor in $\alpha, x_{i}$ is a limit point of some countable subsequence of $\alpha$ running through the terms of $\alpha$ preceding $x_{i}$ in $\alpha$, and (2) $U_{x_{1}}, U_{x_{2}}, U_{x_{3}}, \cdots, U_{x_{i}}, \cdots$ is a monotone descending sequence of distinct open subsets of $M$. In a compact metric space (2) is impossible.

THEOREM 1. A homogeneous, hereditarily unicoherent, compact metric continuum $M$ is indecomposable.

Proof. ${ }^{3}$ Suppose that $U$ is an open subset of $M$ and $H$ is a subset of $M-U$ such that in order for a point $x$ to belong to $H$ it is necessary and sufficient that $U_{x}=U$. In case $M$ contains no such sets $U$ and $H$, $M$ is indecomposable by Theorem 9 of [7].

It is rather easy to see that $H$ is closed. Suppose that there exists a point $y$ of $\bar{H}-H$. Let $z$ be a point of $U_{y}$. Then $M$ is aposyndetic at $z$ with respect to $y$ and hence $M$ is aposyndetic with respect to some point of $H$. Consequently $z$ belongs to $U$. But by the lemma $U_{y}$ cannot be a proper subset of $U$ and hence $U_{y}=U$ and $y$ belongs to $H$. So $H$ is closed.

If $w$ is a point of $M$ such that some point $x$ of $H$ cuts $w$ from a point $z_{1}$ of $U$, then $x$ cuts $w$ from all points of $U$ and $w$ belongs to $H .^{4}$ For suppose that $z_{2}$ is a point of $U$. There exist continua $K_{1}$ and $K_{2}$ and open sets $V_{1}$ and $V_{2}$ such that $M-x \supset K_{i} \supset V_{i} \supset z_{i}(i=1,2)$. Now if $x$ cuts $V_{1}$ from $V_{2}$, it follows from the homogeneity of $M$ that every point of $M$ cuts between two open subsets of $M$; but by Corollary 2 of [8], this is impossible. So $x$ does not cut $V_{1}$ from $V_{2}$ and hence there exists a continuum $K$ in $M-x$ such that $K \cdot V_{1} \neq 0$ and $K \cdot V_{2}$ $\neq 0$. The continuum $K_{1}+K+K_{2}$ contains $z_{1}$ but not $x$; hence $K_{1}+K$ $+K_{2}$ does not contain $w$; consequently $x$ cuts $w$ from all points $z_{2}$ of $U$ and furthermore $z_{2}$ belongs to $U_{w}$. This shows that $U$ is a subset of $U_{w}$ and, by the lemma, $U=U_{w}$. So $w$ belongs to $H$.

${ }^{3}$ Throughout this proof $M$ will be considered to be space. If there do not exist points $x$ and $z$ of $M$ such that $M$ is aposyndetic at $z$ with respect to $x$, then $M$ is indecomposable (Theorem 9 of [7]). So because $M$ is homogeneous it will be assumed that for each point $x$ of $M, U_{x}$ exists (that is, nonvacuous).

4 A point $x$ cuts $w$ from $z$ (in $M$ ) provided that there exists no subcontinuum of $M$ lying in $M-x$ and containing $w+z$. 
For each point $o$ of $H$, let $N_{o}$ denote $o$ together with all points $x$ of $H$ such that $x$ cuts $o$ from $U$. The set $N_{o}$ is closed. Now suppose that for some point $o$ of $H, o$ does not cut all other points of $N_{o}$ from $U$. Then $N_{o}$ contains a point $o_{1}$ such that $N_{o_{1}}$ is a subset of $N_{o}-o$. A homeomorphism of $M$ onto itself carrying $o$ into $o_{1}$ leaves $U$ invariant and carries $o_{1}$ into a point $o_{2}$ of $H$ such that $N_{o_{2}}$ is a proper subset of $N_{o_{1}}$. As in the proof of the lemma, this process may be continued uncountably many times to produce an uncountable monotone sequence of distinct closed sets. This is impossible. Consequently $o$ cuts all other points of $N_{o}$ from $U$. It follows at once that each point of $N_{o}$ cuts all other points of $N_{o}$ from $U$ and in particular if a point $p$ of $H$ cuts a point $o$ of $H$ from $U$, then $o$ cuts $p$ from $U$, and $N_{o}=N_{p}$.

The set $H$ contains no domain. ${ }^{5}$ For suppose on the contrary that $H$ contains a domain $D$. Let $o$ denote a point of $H$. Then $N_{o}$ does not contain $D$, for if it did, a point $x$ of $D$ could cut the domain $D-x$ from the domain $U$ contrary to Corollary 2 of [8]. So $D-D$ - $N_{o}$ is a domain in $H$ containing no point of $N_{o}$. Now $M$ is not aposyndetic at any point of $D-D \cdot N_{o}$ with respect to a point of $N_{o}$. Hence by Theorem 6 of [7], if $z$ is a point of $U, D-D \cdot N_{o}$ contains a point $x$ and $N_{o}$ contains a point $y$ such that $y$ cuts $x$ from $z$ and hence from $U$. Therefore $y$ cuts $x$ from $U$ and consequently $x$ belongs to $N_{o}$. This is a contradiction since $x$ belongs to $D-D \cdot N_{o}$. So $H$ contains no domain.

The domain $U$ is dense in $M$. Suppose the contrary. There exists a domain $D$ lying in $M-(\bar{U}+H)$. Let $y$ be a point of $H$. By the definition of $U, M$ is not aposyndetic at any point of $D$ with respect to $y$. Let $z$ be a point of $U$. By Theorem 6 of [7], $D$ contains a point $x$ such that $y$ cuts $x$ from $z$. Hence (by paragraph 3 of this proof) $x$ belongs to $H$ contrary to construction. So $U$ is dense in $M$ and the boundary of $U$ is $M-U$.

The set $M-U$ is a continuum. Obviously $M-U$ is closed. Suppose that $M-U$ is not connected; then $M-U=A+B$ where $\bar{A}=A$, $\bar{B}=B$, and $A \cdot B=0$. Suppose that $A$ contains a point $x$ of $H$. There exists a domain $D$ such that $D$ contains $B$ but $\bar{D} \cdot A=0$. Each point of the boundary $\beta$ of $D$ belongs to $U$; so there exist a finite collection $K_{1}, K_{2}, \cdots, K_{n}$ of continua and a collection $V_{1}, V_{2}, \cdots, V_{n}$ of domains such that $V_{1}, V_{2}, \cdots, V_{n}$ covers $\beta$ and for each $i, 1 \leqq i \leqq n$, $M-x \supset K_{i} \supset V_{i}$. Since by Corollary 2 of [8] (and the homogeneity of $M$ ) $x$ does not cut any two domains from each other, there exists a

\footnotetext{
5 An open subset of $M$ is called a domain. A domain is not necessarily connected as used here.
} 
continuum $K$ in $M-x$ which contains $D$. Hence $M$ is aposyndetic at each point of $B$ with respect to $x$. This is contrary to the definitions of $B$ and $U$. Hence $M-U$ is connected.

Let $o$ be a point of $H$. Then $N_{o}=M-U$. Suppose on the contrary that $q$ is a point of $M-U$ not in $N_{o}$. If $q$ cuts $o$ from a point of $U_{q}$, then $q$ cuts $U_{q}$ from $o$. Let $T$ be a homeomorphism of $M$ onto itself carrying $o$ into $q .{ }^{6}$, Evidently $T(U)=U_{q}$ and (by paragraph $3, T(H)$ taking the role of $H$ ) $o$ belongs to $T(H)$. Therefore $o$ cuts $q$ from $U_{q}$. But $U_{q} \cdot U \neq 0$ since both $U$ and $U_{q}$ are open, dense subsets of $M$; so $o$ cuts $q$ from a point of $U$. Hence $q$ belongs to $H$. It follows that $q$ cuts $o$ from $U$ and thus belongs to $N_{o}$. From this contradiction it is evident that no point $q$ of $M-\left(U+N_{o}\right)$ cuts $o$ from a point of $U_{q}$. Now let $K$ be a continuum containing a domain $V$ of $U$ and lying in $M-o$. Since each point of $N_{o}$ cuts every other point of $N_{o}$ from $U$, $K$ contains no point of $N_{o}$. Since no point of $N_{o}$ cuts a point $q$ of $M$ $-\left(U+N_{o}\right)$ from a point of $U, K$ may be assumed to contain a point of $M-\left(U+N_{o}\right)$. For each point $q$ of $K \cdot\left[M-\left(U+N_{o}\right)\right]$ there exists a continuum $C_{q}$ from $V$ to $o$ lying in $M-q\left(V \cdot U_{q} \neq 0\right)$. Let $F$ denote a finite collection of these continua, $C_{q}$, such that if $p$ is a point of $K \cdot\left[M-\left(U+N_{o}\right)\right]$, some element of $F$ lies in $M-p$. Suppose that some two continua $C_{q}$ and $C_{t}$ of $F$ intersect in a continuum $C$ containing no point of $K$. Then $C_{q}+K$ and $C_{t}+K$ are continua whose intersection is $C+K$ which is not a continuum. Since $M$ is hereditorily unicoherent, this is a contradiction. Hence $C$ contains a point of $K$. Because $M$ is hereditarily unicoherent, the same reasoning holds when we suppose that $C$ is the common part of all elements of $F$. In this case $C \cdot K$ contains no point of $M-U$ and hence is a subset of $U$. Then $(M-U)+C$ and $(M-U)+K$ are continua whose common part is $(M-U)+C \cdot K$ which is not connected. So $N_{o}=M-U$.

Thus $H$ is a continuum; $H$ is the boundary of $U ; U+H=M$; and every point of $H$ cuts every other point of $H$ from $U$. Let $G$ be a collection consisting of $H$ together with every image of $H$ under homeomorphisms of $M$ onto itself. It is easy to see that $G$ fills up $M$ and no two elements of $G$ have a point in common. Furthermore, $G$ is upper-semicontinuous for if some sequence $x_{1}, x_{2}, x_{3}, \cdots$ of points of distinct elements of $G$ converged to a point $x$ of an element of $G$, say $H$, but some infinite sequence $y_{1}, y_{2}, y_{3}, \cdots$ of points from the same elements of $G$ converged to a point $y$ of $M-H$, then $M$ would be aposyndetic at $y$ with respect to $x$. But for each $i, M$ is not

${ }^{6}$ Roughly stated the purpose of $T$ is merely to shift the frame of reference from $o$ to $q$, so that results already obtained for $H$ and $U$ will apply to similar sets constructed for $q$. 
aposyndetic at $y_{i}$ with respect to $x_{i}$, and this contradicts Theorem 1 of [7]. So $G$ is upper-semicontinuous.

With respect to its elements as points, $G$ is a continuum $M^{\prime}$. Furthermore $M^{\prime}$ is homogeneous and aposyndetic. In such a continuum the meaning of "cut point" and "separating point" are the same [9]. Since $M^{\prime}$ contains a nonseparating point, every point of $M^{\prime}$ is a nonseparating point because of the homeogeneity. Let $A$ and $B$ denote distinct points of $M^{\prime}$ and let $T$ denote a continuum in $M^{\prime}$ irreducible from $A$ to $B$. Let $X$ denote a point of $T-(A+B)$. There exists in $M^{\prime}-X$ a continuum $T_{1}$ containing $A+B$. But $M^{\prime}$ is hereditarily unicoherent. So $T \cdot T_{1}$ is a subcontinuum of $T$ containing $A+B$ but not $X$. This is a contradiction and from this contradiction Theorem 1 follows.

ThEOREM 2. If $M$ is a homogeneous, bounded, plane continuum which does not separate the plane, $M$ is indecomposable.

Theorem 2 follows immediately from Theorem 1 .

The following question remains unanswered: Is every homogeneous, bounded, nondegenerate, plane continuum which does not separate the plane a pseudo-arc?

\section{BibLIOGRAPHY}

1. Stefan Mazurkiewicz, Sur les continua homogènes, Fund. Math. vol. 5 (1924) pp. 137-146.

2. F. B. Jones, A note on homogeneous plane continua, Bull. Amer. Math. Soc. vol. 55 (1949) pp. 113-114.

3. H. J. Cohen, Homogeneous plane continua, Bull. Amer. Math. Soc. Abstract 55-7-422.

4. R. H. Bing, A homogeneous indecomposable plane continuum, Duke Math. J. vol. 15 (1948) pp. 729-742.

5. E. E. Moise, $A$ note on the pseudo-arc, Trans. Amer. Math. Soc. vol. 64 (1949) pp. 57-58.

6. R. L. Moore, Concerning indecomposable continua and continua which contain no subsets that separate the plane, Proc. Nat. Acad. Sci. U.S.A. vol. 12 (1926) pp. 359363, Theorem 4.

7. F. B. Jones, Concerning non-aposyndetic continua, Amer. J. Math. vol. 70 (1948) pp. 403-413.

8. R. H. Bing, Some characterizations of arcs and simple closed curves, Amer. J. Math. vol. 70 (1948) pp. 497-506.

9. G. T. Whyburn, Semi-locally-connected sets, Amer. J. Math. vol. 61 (1939) pp. 733-749, Theorem 6.21.

The University of North Carolina 